\title{
Sheep Infection by Haemonchus Species: Effect on Haematocrit and Evaluation of the FAMACHAC Method in Arsi Negele District, Oromia, Ethiopia
}

\author{
Guash Abay $^{1,}$, , Tilaye Demissie ${ }^{2}$, Amene Fekadu $^{3}$, Teshale Teklue ${ }^{4}$ \\ ${ }^{1}$ Abergelle Agricultural Research Center, Abyi-Adi, Ethiopia \\ ${ }^{2}$ Addis Ababa University, Addis Ababa, Ethiopia \\ ${ }^{3}$ Hawassa University, Hawassa, Ethiopia \\ ${ }^{4}$ Mekelle Agricultural Research Center, Mekelle, Ethiopia
}

Email address:

guashaba81@gmail.com (G. Abay)

\section{To cite this article:}

Guash Abay, Tilaye Demissie, Amene Fekadu, Teshale Teklue. Sheep Infection by Haemonchus Species: Effect on Haematocrit and Evaluation of the FAMACHAC Method in Arsi Negele District, Oromia, Ethiopia. Animal and Veterinary Sciences.

Vol. 3, No. 2, 2015, pp. 74-79. doi: 10.11648/j.avs.20150302.17

\begin{abstract}
Infection with Haemonchus species in sheep at Arsi- Negele district was determined by differential third-stage larvae and the objective was to evaluate the validation of FAMACHAC chart in the diagnosis of Haemonchus infection. The animal's anemia level was determined by using the FAMACHAC method and their corresponding PCV (packed cell volume) were determined by haematocrit centrifugation method. The color of the ocular membrane of all animals was scored 1 to 5 using the FAMACHAC card and blood samples were collected from each animal for determination of PCV. Fecal samples also collected from sampled animals tested for fecal egg counts (FEC), and fecal culture for identification of third stage larvae of Haemonchus species. All sheep studied were negative for Fasciola and were not heavily infested by lice and ticks. A highly significant negative correlations $[(\mathrm{R}$-square $=0.28$, Coef. $=-0.78)$; $(\mathrm{R}$-square $=0.58$, Coef $=-0.34)]$ was observed between Haemonchus larvae and PCV, and PCV and eye color respectively. Positive correlations [(R-square=0.19, Coef. = 1.53); $(\mathrm{R}-$ square $=0.274$, Coef $=0.05)]$ was observed between FAMACHAC score and Haemonchus infection, and FAMACHAC score and egg per gram of faces (EPG) respectively. The PCV of the Haemonchus positive sheep had a strong negative correlation with the EPG (R-square=0.53, Coef $=-0.023$ ). We evaluated the validity of FAMACHAC eye scoring to measure severely infested sheep and got strong correlation among FAMACHAC eye scoring, PCV and Fecal culture positivity. As a conclusion the FAMACHA(C anaemia scoring guide can be used to treat only severely anemic sheep and hence reduce mass treatment and this reduce chance of drug resistance.
\end{abstract}

Keywords: Arsi-Negele, FAMACHA, FEC, Haemonchus, PCV, Sheep

\section{Introduction}

There are about 25.5 million sheep and 24.06 million goats in the country playing an important role in the livelihood of resource-poor farmers [1]. Unlike the large potential of small ruminants in the country their productivity is low. The major constraints of small ruminant production in Ethiopia are diseases, feed shortage and poor management.

Helminthosis has nationwide distribution and is also considered as one of the major setbacks to livestock incurring huge indirect and direct losses in the country [2]. A loss of US $\$ 81.8$ million is reported annually due to helminth parasites in Ethiopia [3]. The blood feeding nematode,
Haemonchus species, is among the most important gastrointestinal parasite of sheep. This parasite, besides its, pathologic significance has a very important position from epidemiological point of view in that it is a parasite of tropical, subtropical and temperate regions of the world [4].

The control of gastro-intestinal parasites of livestock in Ethiopia is based on only the use of anthelmintics. Anthelmentics are imported in bulky by the government, individuals and privates, and distributed all over the country and used voraciously by every individual including farmer. The use of anthelmintics at regular intervals for an extended 
period and treating by mass when one animal shows any clinical sign has resulted in the development of resistance of nematodes to one or more available drugs, for instance against benzimidazoles [5]. There is no field diagnostic technique introduced in Ethiopia but the FAMACHA(C) system can help in the reduction of drug resistance by treating those only unable to cope with current Haemonchus species challenge on pasture, by using clinical anaemia as the determinant. Although FAMACHAC is a novel system of treating only animals with clinical signs and then reducing the chance of development of drug resistance it was checked in developed countries like South Africa, and U.S.A at bigger farm levels and comparatively at net environment, it was not practiced in countries like Ethiopia where mixed parasitc infection, and where traditional feeding practice is followed. So the objectives of this work were to evaluate the effect of haemonchosis on haematocrit value and eye mucous membrane for decision making of individual animal treatment and evaluation of the importance of the FAMACHAC system in Ethiopia in field diagnosis.

\section{Material and Methods}

\subsection{Study Area Description}

The study was carried out in and around Arsi-Negele district peasant associations found around Arsi-Negele town from November 2011 to April 2012. The district is located at an altitude of 1500 to 2300 meters above sea level in the longitude $7^{0} 20^{\prime} \mathrm{N}$ and latitude of $38^{\circ} 40^{\prime} \mathrm{E}$. The district has an estimated 379,645 , cattle population, 111,062 sheep, 151,353 goats, 14,114 horses, 1,455 mules, 27,971 donkeys, 103,206 poultry and 3,450 bee hives [6].

\subsection{Study Animals}

Privately owned sheep flocks were included in the study. The flocks were taken out in the morning for grazing on communal land, road sides and brought back to their holding in the evening. The small ruminants were grazed together with cattle in the open spaces. Different sheep flocks were visited on weekly basis during which 400 sheep (227 Female and 173 Male) were randomly selected from which sample has been collected. Animals free of Fasciola, tick and lice infestation based on the faecal examination and inspection respectively were included in this study.

\subsection{Study Design and Sampling Technique}

A cross-sectional study was followed and animals were randomly selected. Sex, age, eye mucous membrane and body condition of randomly selected animals was recorded. The eye mucous membrane color was compared to the FAMACHA $\subset$ card and was recorded, and $5 \mathrm{ml}$ blood was collected from the jugular vein in vacutainers with anticoagulant (EDTA) after preparing the sites. During sampling general information related to grazing, housing, breeding condition, deworming practice and other factors were collected for each sampled animal.

\subsection{Sample Size Determination}

To maximize the number of animals and to evaluate the system at the prevalence level the sample size was determined using the formula given by [7]:

$$
N=\frac{1.96^{2}(P)(1-P)}{d^{2}}
$$

Where; $\mathrm{N}=$ sample size, $1.96=$ the value of $\mathrm{z}$ at $95 \%$ confidence level, $\mathrm{P}=$ expected prevalence, $\mathrm{d}=$ desired absolute precision level at $95 \%$ confidence level

Expected prevalence of $50 \%$ were considered as there was no previous study in the area and 5\% absolute precision, the minimum sample size calculated was 384 . Therefore about 400 sheep of all age group were selected from study sites.

\subsection{Sample Collection}

\subsubsection{Blood Sample Collection}

Blood samples were taken in EDTA coated vacutainer tubes from the jugular vein to determine PCV. The blood samples were taken to the laboratory of veterinary parasitology and pathology, school of veterinary medicine and PCV were determined immediately after arrival using the micro-haematocrit method.

\subsubsection{Fecal Sample Collection}

Fecal samples were collected per-rectum using a disposable plastic gloves in universal while blood sample collection and labeled and kept cool before transportation to the laboratory using ice box on the same day of collection and were processed immediately.

The positive samples were subjected to fecal culture for investigating the infective larvae. Fecal sample that were positive Fasciloa egg were discarded.

\subsection{Laboratory Investigation}

\subsubsection{Packed Cell Volume Determination}

At the laboratory PCV was determined and the level of anemia was recorded for each animal according to [8].

\subsubsection{Egg per Gram of Faeces Determination}

A modified McMaster technique was used to determine fecal egg counts according to [9].

\subsubsection{Ova Culture}

After screening for Fasciola and FECs the feces were cultured for $\mathrm{L}_{3}$ of Haemonchus species at a temperature of approximately $25^{\circ} \mathrm{c}$ according to [9].

\subsubsection{Identification of Haemonchus Species Third Stage Larvae}

The identification was conducted based on the distinguishing features such as the shape of the "head" (cranial extremity) of the larvae or the length of the sheath "tail" (the extension of the sheath from the tip of the larval caudal extremity to the tip of the tail of sheath) [10]. 


\section{Data Analysis}

Data were subjected to stata-11 software. Correlations between PCV and eye mucous membrane color, PCV and FEC, and eye mucous membrane color and FEC were compared at $\mathrm{p}<0.05$.

\section{Result}

Out of the 400 fecal cultured 151(37.75\%) were purely positive for Haemonchus and 52 were positive for Haemonchus and other nematodes. Based on results of faecal cultures, Haemonchus species was the primary gastrointestin al nematode infecting sheep.

\subsection{Effect of Haemonchus Larvae on Packed Cell Volume}

In the present study the minimum observed PCV was $10 \%$ and the maximum $43.5 \%$ with the mean PCV $24 \pm 0.43 \%$. A highly significant negative correlation $\left(\mathrm{R}^{2}=0.28\right.$, Coef. $=$ $0.78, \mathrm{P}<0.001)$ was observed between Haemonchus larvae and PCV as indicated in Table 1. Many lower haematocrit values (PCV) were registered during periods of heavier Haemonchus infection that is when many animals were positive and the lowest PCV values were also recorded for Haemonchus positive animals than other nematode positive animals. That means more animals with Haemonchus positive were identified with lower haematocrit (PCV) value than negative animals.

\subsection{Effect of Haemonchus Larvae on Eye Color}

There was significant positive correlation between the eye color score (FAMACHAC score) and Haemonchus infection in the study area as indicated in Table $1\left(\mathrm{R}^{2}=0.19\right.$, Coef. $=$ 1.53, $\mathrm{P}<0.001$. There was a positive correlation between Haemonchus larvae and eye score meaning animals that were positively cultured for Haemonchus showed severe anemia (5 score) and anemia (4 score) compared to animals that were positive to other nematode parasites. During high Haemonchus infection (short rainy season/when many animals were culture positive) the percentage of sheep with FAMACHAC scores of 4 and 5 were increased, and vice versa. Some animals were culture positive for Haemonchus but the FAMACHAC score were score 1, score 2 and/or score 3 (not signs of anemia), this may be due to pre-patent period of infection, or due to low worm burden or hostresistance to GI helminthes. There were also few animals that were Haemonchus negative on culture but with FAMACHAC score fall in 3,4 and 5. This may be due to nutritional deficiencies.

Table 1. Frequency of animals with Haemonchus species positive with ranges of PCV and FAMACHA $\odot$ score in sheep in Arsi-Negele districts.

\begin{tabular}{|c|c|c|c|c|c|c|c|c|}
\hline \multicolumn{3}{|c|}{ PCV values } & & \multicolumn{5}{|c|}{ Eye mucous membrane color } \\
\hline H.larvae & $\mathrm{PCV}<19$ & PCV20-29 & $\mathrm{PCV}>30$ & Ec1 & Ec2 & Ec3 & Ec4 & Ec5 \\
\hline Positive & 54 & 74 & 23 & 37 & 10 & 18 & 21 & 65 \\
\hline negative & 9 & 75 & 142 & 140 & 20 & 20 & 14 & 38 \\
\hline
\end{tabular}

Keys: $\mathrm{Ec}=$ eye color

\subsection{Packed Cell Volume and Eye Color (FAMACHA ${ }^{\circ}$ -} categories)

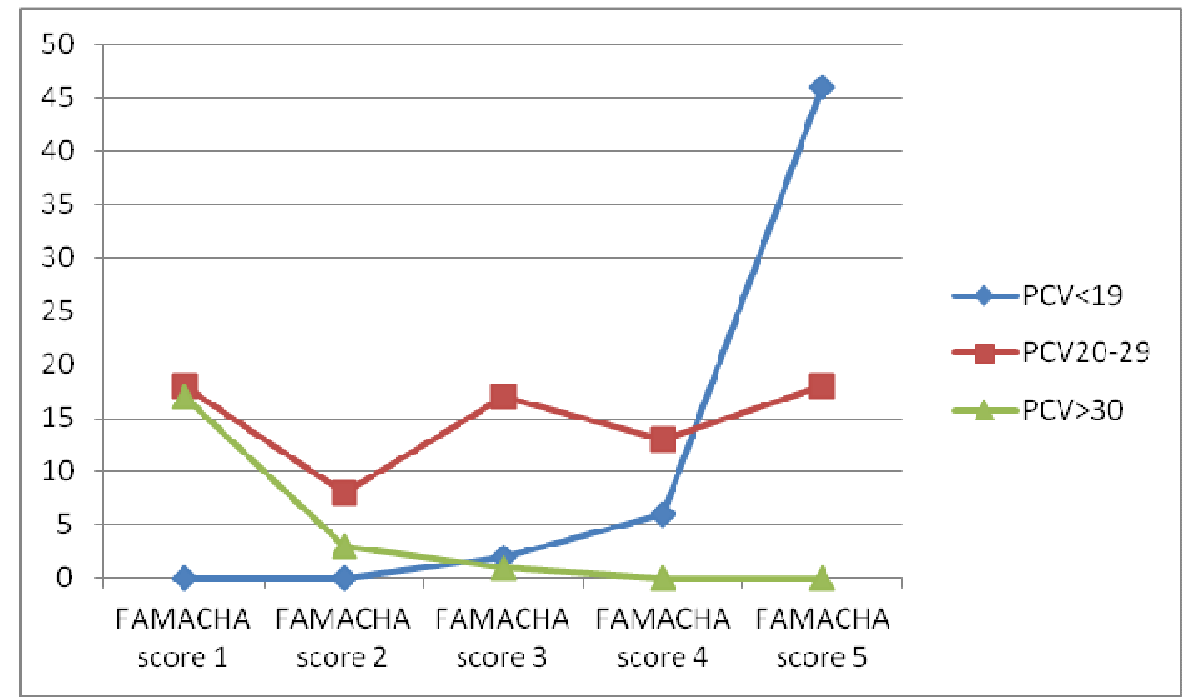

Figure 1. Correlation between PCV and FAMACHA $\odot$ score; indicates that FAMACHAC eye color scoring of 3, 4 and 5 measured many animals with PCV values less than 19

To evaluate the correlation between the amount of red blood cells and the colour of the conjunctiva, the PCV of blood samples and FAMACHAC scores were simultaneously and independently determined. There was an agreement too 
between the lower haemtocrits and paler mucous membrane scored according to FAMACHAC method. The correlations between $\mathrm{PCV}$ and eye color were strong and negative $\left(\mathrm{R}^{2}=\right.$ 0.58 , Coef $=-0.34, \mathrm{P}<0.001$ ) for sheep in the study area as indicated in Fig.1, that is as the eye color score approaches score 5 (severe anemia) the PCV level dropped the lowest values $(\mathrm{PCV}<19)$. The FAMACHAC categories of score 4 and 5 or sometimes category score of 3 were considered positive for anemic animals, and categories one and two or sometimes three, were considered negative.

\subsection{Quantitative Fecal Examination}

The McMaster technique was used to determine the number of EPG revealed an overall mean EPG of 1584 with minimum and maximum count of 50 and 7,450 respectively. There was a significant association between the mean EPG and eye color score.

\subsubsection{Fecal Egg Count and Packed Cell Volume}

There was a strong negative correlation between the PCV of the Haemonchus positive sheep and the EPG $\left(\mathrm{R}^{2}=0.53\right.$, Coef $=-0.023, \mathrm{P}<0.001)$ as indicated in Fig.2. That is, during high EPG the lower PCV value was recorded. Many animals were anemic and severe anemic for Haemonchus positive animals, and EPG of these animals was also higher than animals positive for other nematodes.

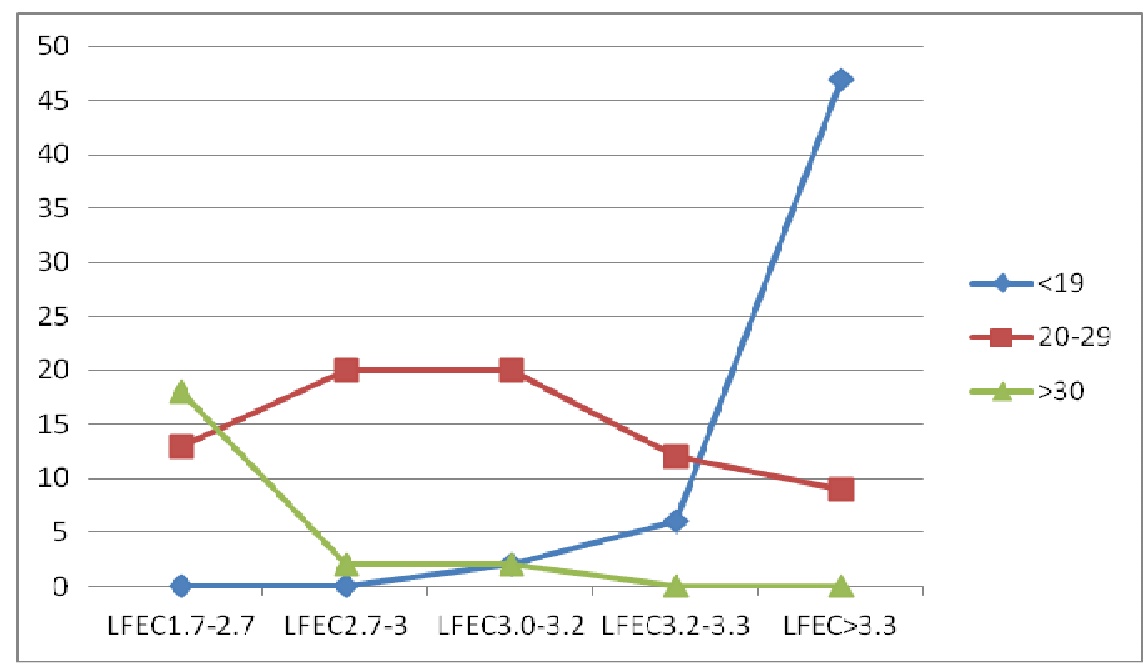

Figure 2. Correlation between PCV and FAMACHA® score; indicates that FAMACHA® eye color scoring of 3, 4 and 5 measured many animals with PCV values less than 19.

\subsubsection{Faecal Egg Count and Eye Color (FAMACHA(C) Categories}

To evaluate the correlation between FAMACHA® scoring and the number of excreted eggs for pure culture positive sheep (culture negative for other nematodes) the FAMACHAC-scores and the EPG were independently recorded.

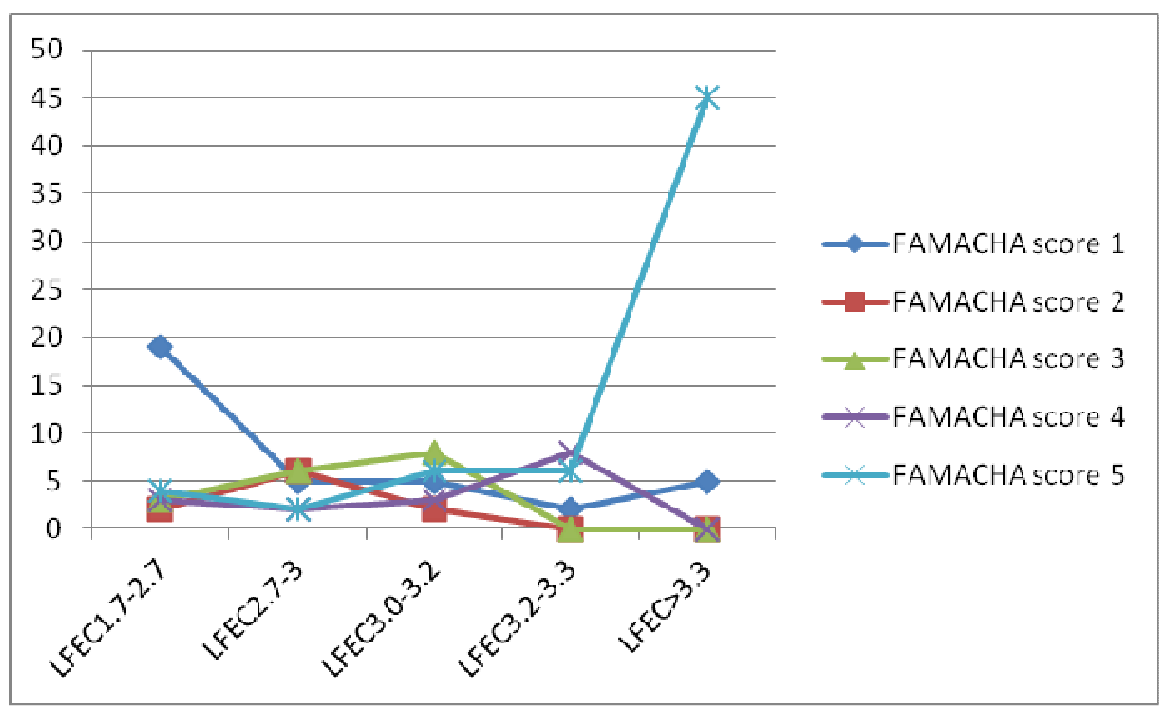

Figure 3. Correlation between EPG and eye color; indicates that LFEC $>3.3$ measures many animals with FAMACHA@ score 5.

For FAMACHA(C) category 3, the EPG ranges was between 1050 EPG and 1500 EPG, for category 4 ranges 
between 1550 EPG and 2000 EPG and for category 5 a FEC was greater than 2000 EPG. The frequencies of FEC categories for each FAMACHAC-score are presented in figure 5. The result elucidated a relationship between EPG level and eye mucous membrane color (FMACHAC score). The FAMACHAC score had a strong positive correlation $(\mathrm{R}=0.274, \mathrm{P}<0.001)$ with EPG as indicated in Fig. 3 i.e. the percentage of sheep with FAMACHA(C) scores of 3, 4 and 5 increased as the EPG for Haemonchus positive animals increased than EPG for other nematodes. Results revealed that in sheep with an EPG level of 1050-2000, most of the animals fell in category 3, 4 with pink and pink white eye color. Similarly, with EPG level above 2000 animals fell in category 5 , having white eye color showing sever anemic condition.

\section{Discussion}

In this study $37.8 \%$ of the 400 animals were positive to Haemonchus infection as to cultured faeces for throughout the study periods and this was in agreement with [10]. In the study area just like other parts of the country antihelmintics were used frequently and almost for animals visiting the clinic. Multiple-drug resistant nematodes especially to Haemonchus species have been reported in many areas of the world and Haemonchus species were known for developing drug resistant $[11,13-15]$ to the extent that total anthelmintic failure is seen as a real possibility in some situations.

In present work the PCV of the study sheep had a strong negative correlation with the FAMACHAC eye colour scores and this agreed with the findings of [16], found that strong negative correlations $\left(\mathrm{R}^{2}=-0.72, \mathrm{P}<0.0001\right)$ for the FAMACHA $\subset$ eye color and PCV. Thus, the FAMACHAC anaemia scoring guide can measure significant number of sheep positive for Haemonchus and anemic and severely anemic and thus can be utilized as procedure for the indirect diagnosis of haemonchosis if other causes of anemia were minimum. In the present study the FAMACHAC score had a strong positive correlation with EPG that is the percentage of sheep with FAMACHAC scores of 3, 4 and 5 increased as the EPG increased, and this was in line with the finding of [17]. [17] States that animals with high FAMACHAC scores generally had high FECs, and vice versa. However, the prolificacy of worms can be affected by many factors such as season and host immunity, so repeated measures should be taken.

When PCV fell below 19\%, FEC were higher than $1050 \mathrm{EPG}$ and the majority of FEC were above 2000EPG and this was agreed with the finding of $[4,5,14,16-18]$. Such an association might allow drawing some conclusions on the intensity of infection with Haemonchus species as Haemonchus species are prolific egg layers than other nematodes.

Targeted or selective application of anthelmintic treatment might be an important tool to keep susceptible GIN strains in livestock and to delay the development of antihelmintic resistance. The accuracy and feasibility of FAMACHAC was tested in 400 sheep in Arsi-Negele districts and such method [19] can be used by the farmers themselves by checking their animals for signs of anaemia.

\section{Conclusion and Recommendations}

The PCV and FECs, FECs and eye mucous membrane color (FAMACHAC score) were significantly associated in the study sheep flock. Based on the results of this work it can be concluded that the FAMACHA(C method can be used as quick, simple and cheap alternative for an integrated control of nematode parasites, particularly when Haemonchus species is the primary pathogen which would result in reducing the frequency of anthelmintic treatments which in turn result in reduced chance of drug resistance. Based on the above the following recommendations are forwarded;

- Still now there are few works on the validation of FAMACHAC chart in Ethiopia so FAMACHAC eye color evaluation that covers the large parts of the country should be done and results should be pooled together, evaluated and broad conclusions should be drawn on the use of FAMACHAC system.

- There was agreement too between the lower haematocrits and paler mucous membranes scored according to the FAMACHAC method. The use of this system may be recommended as part of an integrated approach to worm control in sheep kept in the resourcepoor areas studied.

\section{Acknowledgments}

Our acknowledgment goes to all members of Hawassa University School of Veterinary Medicine parasitology laboratory workers for allowed me to use all the available facilities.

\section{References}

[1] CSA, 2013. Central statistics for livestock population in Ethiopia. Addis Ababa, Ethiopia

[2] Kumsa B. and Wossene A. 2006. Abomasal nematodes of small ruminants of Ogaden region, Eastern Ethiopia. Prevalence, worm burden and species composition, Revue Med. Vet, 157:27-32.

[3] Demelash B., Yilma J. and Hassen C. 2006. Ovine helminthosis is major health constraints to productivity of sheep in Ethiopia. Faculty of Veterinary Medicine, Hawassa University, Hawassa, Ethiopia, pp. 65-90.

[4] Chaudary F.R., Khan M.F. and Qayyum M. 2007. Prevalence of Haemonchus contortus in naturally infected small ruminants grazing in the Potohar area of Pakistan. Pakistan vet. J, 27:73-79.

[5] Woldemariam D.L. 2005. Nematode Prevalence, helminth management practices and anthelmintic resistance in small ruminants in the mid rift valley of Ethiopia. University of Pretoria, Faculty of veterinary sciences, Pretoria, South Africa, Pp.2-3, 29, 60. 
[6] Arsi-Negele beureau of agriculture and rural development, 2013.

[7] Thrusfield M. 2008. Sampling in Veterinary epidemiology. $2^{\text {nd }}$ ed, Blackwell science, London. Pp.198

[8] Hansen J. and Perry B. 1994. The epidemiology, diagnosis and control of helminth parasites of ruminants. $2^{\text {nd }} \mathrm{ed}$, Nairobi, Kenya: ILRAD (international laboratory for research on animal diseases).

[9] Hendrix C.M. and Robinson E. 2012. Diagnostic parasitology for veterinary technicians. $4^{\text {th }}$ ed, Mosby, USA, Pp.54.

[10] MAFF (Minstry of Agriculture, Fisheries and Food), 1997. Manual of Veterinary parasitological laboratory techniques. Tech.Bull, NO. 18, Her Majesty's stationery office, London, Pp.129.

[11] Zeryhun T. 2011. Helminthosis of sheep and goats in and around Haramaya, Southeastern Ethiopia. Journal of Vet. Med. and Animal Health, 4:48-55.

[12] Burke J.M., Kaplan R.M., Miller J.E., Terrill T.H., Getz W.R., Mobini S., Valencia E., Williams M.J., Williamson L.H. and Vatta A.F. 2007. Accuracy of the FAMACHAC system for onfarm use by sheep and goat producers in the southeastern United States. Vet. Parasitol, 147:89-95.

[13] Chandrawathani P., Yusoff N., Wan L.C., Ham A. and Waller P.J. 2004. Total anthelmintic failure to control nematode parasites of small ruminants on government breeding farms in Sabah East Malaysia. Vet. Res. Comm, 28: 1-11.
[14] Kaplan R.M., Burke J.M., Terrill T.H., Miller J.E., Getz W.R., Mobini S., Valencia E., Williams M.G., Williamson L.H., Larsen M. and Vatta A.F. 2004. Validation of the FAMACHC eye color chart for detecting clinical anemia in sheep and goats on farms in the Southern United States. Vet. Parasitol, 123: $105-120$

[15] Kumsa B. 2004. Study on Ogaden small ruminant haemonchosis: morphological characterization and susceptibility to Albendazole and Tetramisole. Msc thesis, school of veterinary medicine, Addis Ababa University, Addis Ababa, Ethiopia.

[16] Menkir S. 2007. Helminth Parasites of Sheep and Goats in Eastern Ethiopia: Epidemiology, and Anthelmintic Resistance and its Management. Doctoral thesis, Faculty of Veterinary Medicine and Animal Science, Swedish University of Agricultural Sciences, Uppsala, Sweden.

[17] Scheuerle M. 2009. Anthelmintic resistance of Haemonchus contortus and the FAMACHAC method as a tool to delay the development of anthelmintic resistance. $1^{\text {st }}$ ed. vvb laufersweiler verlag, pp.4.

[18] Saddiqi H. 2010. Evaluation of some indigenous breeds of sheep for natural resistance against Haemonchus contortus infection. Doctoral thesis, Faculty of Veterinary science, University of Agriculture Faisalabad, Pakistan.

[19] VanWyk J.A. 2001. Refugia - overlooked as perhaps the most potent factor concerning the development of anthelmintic resistance. Onderstepoort J. Vet. Res, 68: 55-67. 